\title{
Evaluación de la deglución con nasofibroscopia en pacientes hospitalizados: factores predictivos y seguimiento intrahospitalario. Experiencia en un hospital clínico universitario
}

\author{
RODRIGO CABEZÓN A., CONSTANZA RAMÍREZ R. ${ }^{a}$, PEDRO BADÍA V., \\ NORMA LEÓN M. ${ }^{\mathrm{b}}$, XIMENA FONSECA A.
}

\section{Functional endoscopic evaluation of swallowing among hospitalized patients}

Background: Swallowing disorders are common in hospitalized patients. Functional endoscopic evaluation of swallowing (FEES) is a simple, safe and effective test for the diagnosis and monitoring of these disorders. Aim: To report the results of functional endoscopic swallowing evaluation. Material and Methods: Retrospective review of all records of functional swallowing evaluation procedures performed in a 6 months period. Results: A total of 327 evaluations were analyzed. Sixty seven percent were performed for the first time in a patient and $32.4 \%$ were follow-up evaluations. Mean age of patients was $73 \pm 17$ (SD) years and 56\% were male. Twenty nine percent had prolonged orotracheal intubation ( $>48$ hours). Swallowing was evaluated as normal, mildly, moderately and severely altered in 8.2,27, 27 and 38\% of cases, respectively. Age ( $>50$ years) and orotracheal intubation were the most important predictors of severity $(p=0,01)$. Oral feeding was achieved during hospital stay in 78 and $55 \%$ of patients with moderate or severe swallowing disorders, respectively, after a delay of 8.7 and 14.3 days, respectively. Having a severe swallowing disorder during the first evaluation, increased fourfold the risk of gastrostomy. Conclusions: Most hospitalized patients with swallowing disorders achieve oral feeding before leaving hospital. Safe oral feeding is delayed as swallowing disorder is of greater severity.

(Rev Med Chile 2011; 139: 1025-1031).

Key words: Deglutition disorders; dysphagia; Intubation, gastrointestinal.

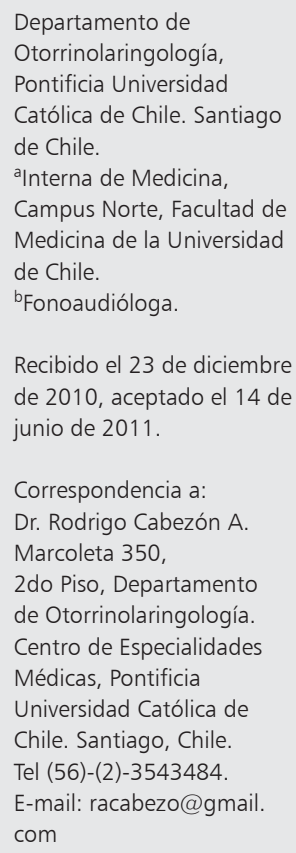

$\mathrm{D}$ isfagia es cualquier alteración en el avance de la comida desde la boca al estómago. Una definición más estructurada es la acuñada por Johnson"; "Trastorno para tragar alimentos sólidos, semisólidos o líquidos por una deficiencia en cualquiera de las 4 etapas de la deglución (preparatoria oral, oral, faríngea y esofágica), y que puede ocasionar neumonía, desnutrición, deshidratación y obstrucción de la vía aérea". Se asocia a las personas de mayor edad, alcanzando hasta $60 \%$ de los adultos mayores hospitalizados². La neumonía aspirativa es la mayor morbilidad asociada a esta condición, ella aumenta considerablemente la estadía hospitalaria y sus costos asociados, presentando hasta $40 \%$ de mortalidad, por lo cual su prevención y efectiva rehabilitación permite mejorar los resultados globales de atención ${ }^{3,4}$. 
La incidencia de trastornos de la deglución está en aumento en Chile y el mundo, entre otras causas por el aumento sostenido de la población mayor de 65 años $(7,9 \%$ de la población chilena en el 2005) $)^{5}$, la mayor presencia de comorbilidades asociadas, y la mejor sobrevida de patologías que provocan secuelas, como el accidente vascular encefálico (AVE), que pueden presentar disfagia hasta en $80 \%$ de los casos, dependiendo del método diagnóstico utilizado ${ }^{6-8}$. Se ha descrito también la mayor frecuencia de trastornos de la deglución en pacientes con antecedente de intubación prolongada previa ${ }^{9-11}$. La Tabla 1 resume las causas de disfagia orofaríngea.

La deglución es un acto neuromuscular de alta complejidad, que involucra procesos voluntarios e involuntarios, que se realiza alrededor de 1.000 veces al día. Se ha postulado que el nervio laríngeo superior (rama del nervio vago) es el principal encargado de la sensibilidad laríngea y sería el mayor responsable en el gatillo del reflejo de la

Tabla 1. Etiología de las alteraciones de la deglución ${ }^{4}$

\begin{tabular}{|ll|}
\hline Obstructivas & Neoplasia vía aereodigestiva superior \\
& Estenosis esofágica \\
& Acalasia, disfunción cricofaríngea \\
& Compresión extrínseca \\
& Osteofito cervical \\
Enfermedades & Miastenia gravis \\
neurológicas & ELA, Esclerosis múltiple \\
& Parálisis cerebral \\
& Alzheimer, Parkinson \\
& Retardo mental \\
& Distrofia muscular \\
& Síndrome postpolio \\
& Neuropatía de pares craneanos \\
& AVE \\
Daño & TEC \\
neurológico & Neurocirugía \\
& Cirugía de base de cráneo \\
latrogénicas & Intubación \\
& Traqueostomía \\
& Cirugía \\
& Radioterapia cervical \\
Fármacos \\
Misceláneas & Xerostomía \\
& Presbifagia o presbiesófago \\
& Politraumatismo \\
& RGE \\
Divertículo de Zenker
\end{tabular}

deglución. Por lo tanto, el trastorno de la deglución puede ser causado por factores anatómicos, motores, de sensibilidad, o mixtos ${ }^{3}$. Asimismo, debe hacerse la distinción entre disfagias "altas" (orofaríngeas) de las disfagias "bajas", donde debe realizarse derivación al gastroenterólogo para un estudio anatómico y funcional.

Ningún examen es totalmente sensible para el diagnóstico y seguimiento de los pacientes con trastornos de la deglución, debido a la multiplicidad de causas que lo pueden provocar. Dentro de los exámenes disponibles para el estudio de las disfagias orofaríngeas, existe la prueba de agua, la videofluoroscopia (o "trago de bario"), la evaluación foniátrica indirecta, la manometría esofágica (en caso de sospecha de acalasia cricofaríngea), y la evaluación nasofibroscópica de la deglución (FEES, Functional Endoscopic Evaluation of Swallowing), de resorte del otorrinolaringólogo, con la cooperación de fonoaudiología ${ }^{12,13}$. Por esta razón, el examen a utilizar en cada servicio dependerá de la disponibilidad de especialistas y recursos con que cuenta cada centro, considerando también la importancia del seguimiento y la rehabilitación.

Nuestro Departamento sigue desde hace 3 años un protocolo para evaluar la deglución con nasofibroscopia (FEES $)^{14}$, que presenta ventajas comparativas, tales como la evaluación "al lado de la cama" del paciente (sin necesidad de traslado), la ausencia de irradiación (considerar seguimiento del paciente con evaluaciones sucesivas), y la mejor evaluación de la sensibilidad laríngea (Figura 1). Reconocemos algunas limitaciones de nuestro método de evaluación, principalmente la existencia de un tiempo ciego al examinador, la imposibilidad de evaluar la fase esofágica (que puede ser solucionado complementando nuestro examen con otra prueba diagnóstica en caso de sospecha) y, por supuesto, la disponibilidad del equipo y de especialistas ${ }^{15,16}$.

Para realizar nuestra evaluación, solicitamos que el paciente esté estable, y con el mejor estado de conciencia posible (idealmente lúcido y cooperador). Con el paciente en posición sentada, se realiza nasofibroscopia, mientras la fonoaudióloga alimenta al paciente con jalea teñida con azul de metileno, registrando las fases de la deglución, y repitiendo el bolo alimentario si es necesario. Posteriormente, se extiende un informe protocolizado, con las indicaciones sugeridas, así como la 


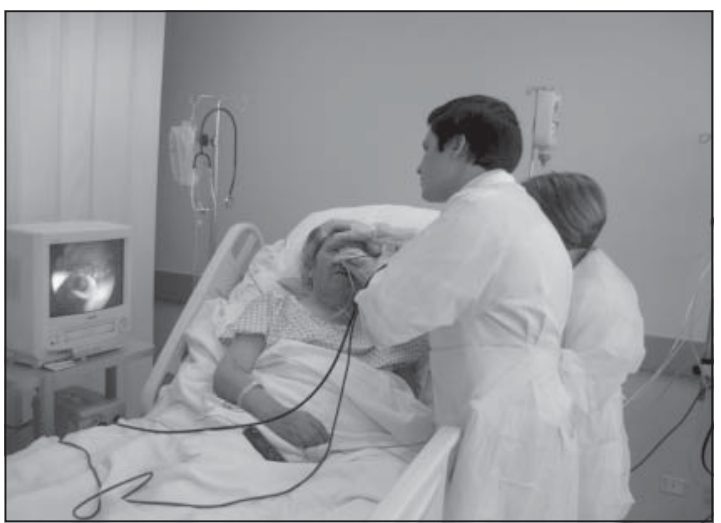

Figura 1. Evaluación de un paciente hospitalizado. Se pueden observar algunas ventajas de esta forma de evaluación, tales como su ejecución directamente en la cama del paciente, sin necesidad de trasladarlo a otro Servicio, y la ausencia de irradiación.

indicación de control posterior según la evaluación por el equipo de rehabilitación foniátrica. La Tabla 2 muestra la clasificación que utilizamos para los trastornos deglutorios según $\mathrm{FEES}^{14}, \mathrm{y}$ en la Tabla 3, nuestro algoritmo para el manejo de los pacientes en quienes se decide postergar la alimentación por vía oral.

Los objetivos del presente estudio son caracterizar a la población evaluada con FEES en pacientes hospitalizados, determinar los factores predictivos más relevantes en la evolución del trastorno deglutorio que se puedan conocer en la evaluación inicial, comparándolos con los descritos en la literatura, y describir la evolución de los pacientes que fueron seguidos en forma completa por nuestro equipo.

\section{Materiales y Métodos}

Se utilizó el mismo protocolo de evaluación de la deglución con nasofibroscopia en todos los pacientes, con presencia de al menos un otorrinolaringólogo y la misma fonoaudióloga en todos los casos. Se clasificó la severidad del trastorno de deglución según lo publicado por Beltrán y cols ${ }^{14}$, en: ausencia de trastorno deglutorio, trastorno leve, moderado o severo, generando un puntaje de 0 a 3 puntos según el grado de severidad registrado (Tabla 2).

Se realizó una revisión retrospectiva de todos los registros de evaluación de deglución (FEES) de pacientes hospitalizados, realizados por el Departamento de Otorrinolaringología del Hospital Clínico de la Pontificia Universidad Católica de Chile, entre el 1 de enero y el 30 de junio de 2010. Se recopilaron los datos demográficos, antecedente de intubación orotraqueal prolongada (definida como aquella mayor a 48 horas), patologías de

Tabla 2. Clasificación de la severidad de los trastornos deglutorios

\begin{tabular}{|llc|}
\hline Clasificación & Puntaje \\
\hline Sin trastorno & $\begin{array}{l}\text { Deglución dentro de límites } \\
\text { normales }\end{array}$ & 0 \\
Leve & $\begin{array}{l}\text { Enlentecimiento o descoordi- } \\
\text { nación de fases I y II }\end{array}$ & 1 \\
\hline Moderado & $\begin{array}{l}\text { Retención persistente de ali- } \\
\text { mentos y/o secreciones en vía } \\
\text { aérea superior } \\
\text { Penetración, sin aspiración } \\
\text { evidente }\end{array}$ & 2 \\
Severo & $\begin{array}{l}\text { Aspiración evidente, con o sin } \\
\text { reflejo de tos }\end{array}$ & 3 \\
\hline
\end{tabular}

Clasificación utilizada en nuestro protocolo de evaluación (penetración: ingreso de alimento y/o secreciones a supraglotis; aspiración: paso de alimentos y/o secreciones bajo la glotis) ${ }^{14}$

Tabla 3. Algoritmo para el manejo en pacientes con trastorno moderado o severo de la deglución

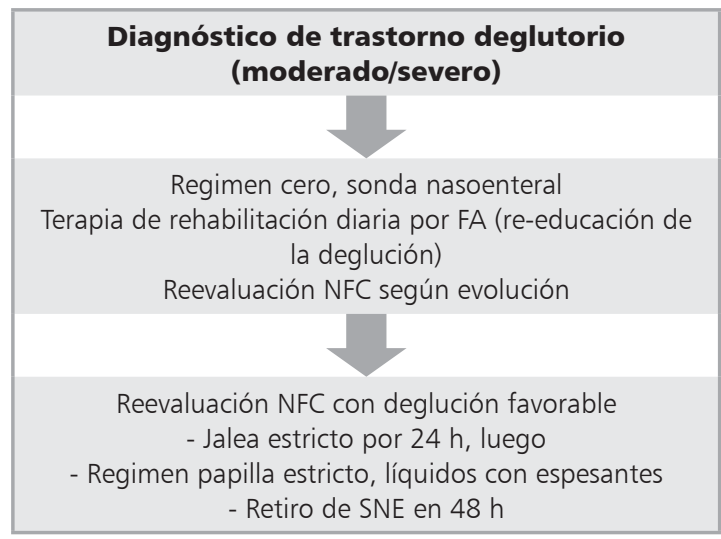

Los pacientes sin trastorno deglutorio son excluidos de protocolo de seguimiento. Los con trastorno leve son autorizados a recibir alimentación por vía oral, supervisados inicialmente por fonoaudiología, sugiriéndose en algunos casos la rehabilitación foniátrica posterior. 
ingreso, severidad del trastorno deglutorio en la primera evaluación, así como el seguimiento con las evaluaciones posteriores en aquellos pacientes que las poseían. Se registró la vía de alimentación al alta sugerida por el equipo evaluador: alimentación por vía oral (VO), egreso con sonda nasoenteral (SNG), o gastrostomía (GTT)). Se registraron los datos en tabla de Excel 2007, y para el análisis estadístico se utilizó el programa Minitab 15.0 en Español, utilizando pruebas de t de Student para muestras independientes, test $Z$, regresión lineal $\mathrm{y}$ análisis multivariado. Se consideró significativo un valor de $\mathrm{p}<0,05$.

\section{Resultados}

De un total de 327 evaluaciones registradas, $221(67,6 \%)$ fueron pacientes nuevos, y 106 $(32,4 \%)$ fueron seguimientos. La edad promedio de los sujetos evaluados fue de 73,2 $\pm 17,26$ años (rango: 16 a 97), existiendo un predominio de pacientes de género masculino (55,7\%). El 29\% de los pacientes presentaba antecedente de intubación prolongada. El resultado de la evaluación de la deglución según severidad se expone en la Figura 2.

La patología de ingreso más frecuente por la cual se solicitó evaluación de la deglución fue el accidente vascular encefálico (AVE), en 33\% de los casos $(\mathrm{n}=72)$, seguido de pacientes con patologías respiratorias (principalmente neumonía en el adulto mayor), $13 \%$, y por pacientes con patología cardiovascular (11\%). La Figura 3 mues-

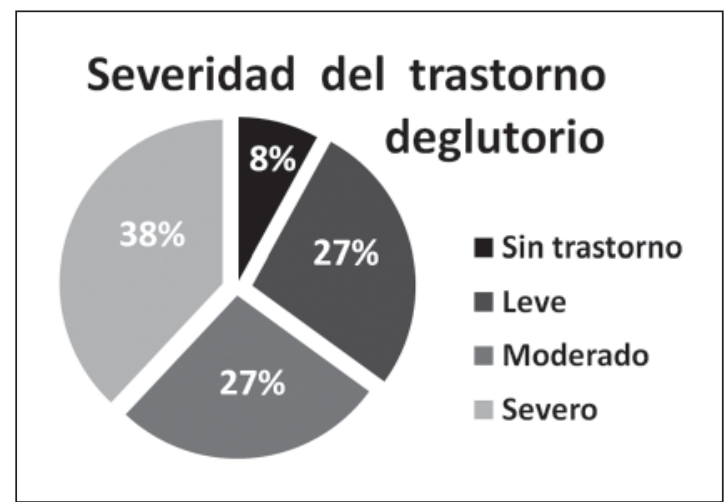

Figura 2. Distribución de la severidad del trastorno deglutorio en los pacientes evaluados (primera evaluación).

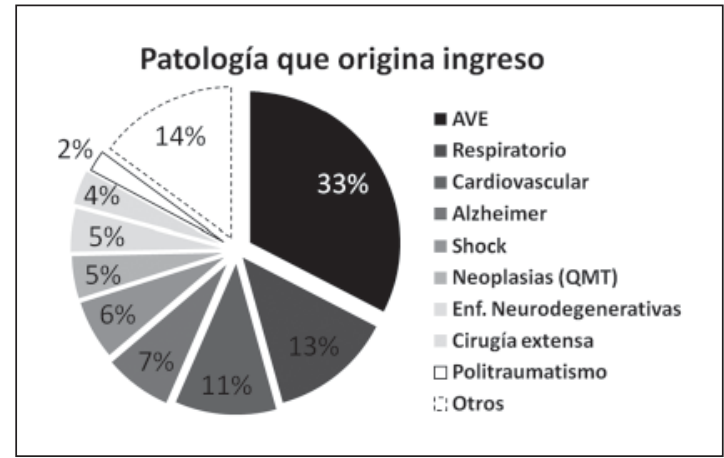

Figura 3. Patologías que originan el ingreso al Hospital de los pacientes evaluados (QMT: Quimioterapia).

tra las patologías de ingreso de los pacientes y el grado de severidad del trastorno deglutorio según patologías se muestra en la Figura 4.

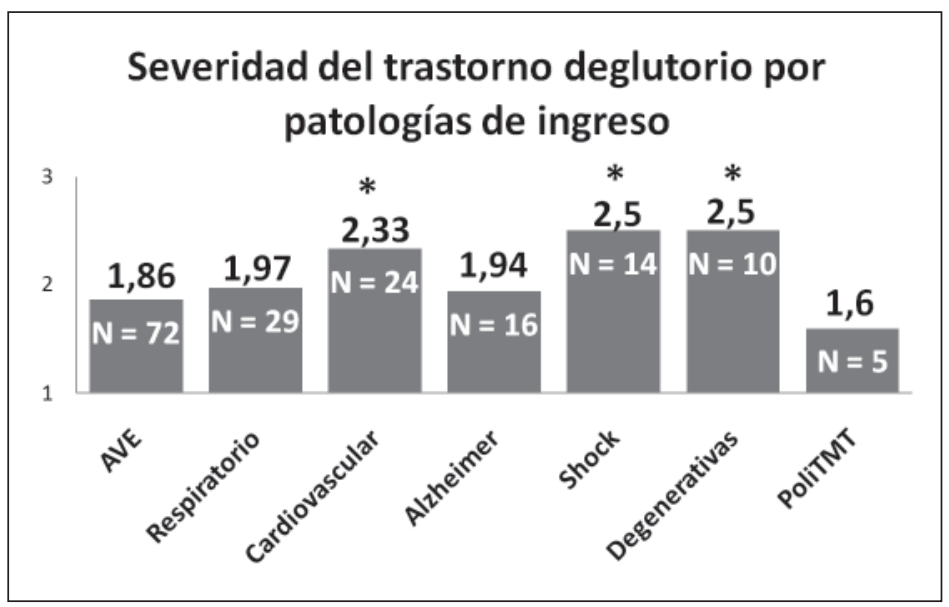

1028 


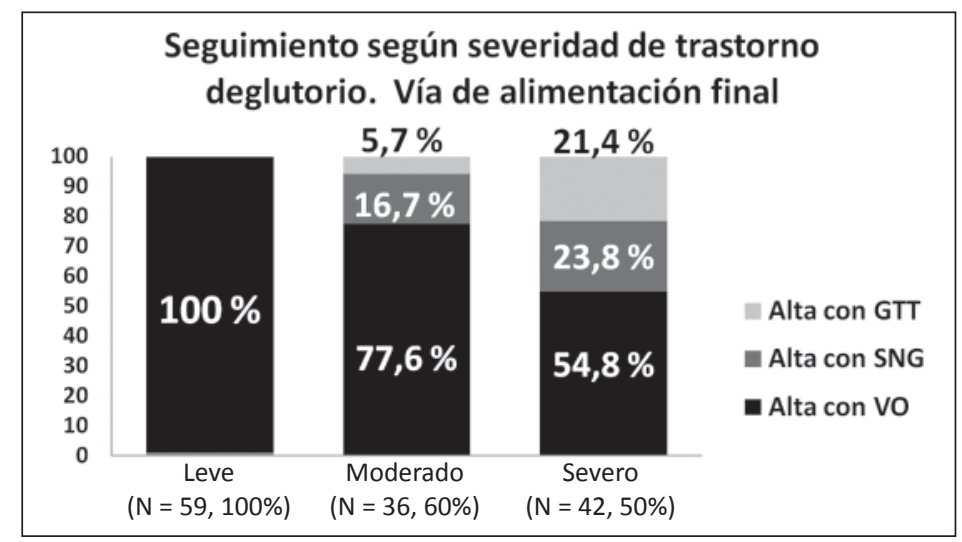

Figura 5. Vía de alimentación al egreso hospitalario. En paréntesis, \% de seguimiento de cada grupo (VO: realimentación por vía oral; SNG: alimentación nasoenteral; GTT: Gastrostomía).
La totalidad de los pacientes sin trastorno detectable o con un trastorno leve de la deglución lograron realimentación por vía oral (VO) antes del alta. Por el contrario, para la mayoría de los pacientes con trastorno moderado y severo de la deglución se decidió la postergación inicial de la realimentación $\mathrm{VO}$, indicándose alimentación nasoenteral, rehabilitación por fonoaudiología, y reevaluaciones seriadas, según evolución. Se logró un seguimiento completo de $60 \%$ de los pacientes con trastorno moderado y de $50 \%$ de los pacientes con trastorno severo de la deglución. La Figura 5 muestra los resultados finales del seguimiento.

\section{Discusión}

Se analizaron los datos de edad, género y antecedente de intubación prolongada previa como predictores del grado de severidad del trastorno deglutorio, usando regresión lineal. Se observó que la intubación previa es el factor predictor más relevante en el grado de severidad del trastorno deglutorio $(\mathrm{R}=18,4 \%, \mathrm{p}=0,006)$, seguido de la edad del paciente $(\mathrm{R}=15,8 \%, \mathrm{p}=0,025)$. El género del paciente no explicaría la severidad del trastorno deglutorio $(\mathrm{R}=2,7 \%, \mathrm{p}=0,48)$.

Los pacientes con antecedente de intubación prolongada tienen una edad promedio menor que los que no tienen este antecedente ( 67 vs 75,8 años, $\mathrm{p}<0,001)$, y son predominantemente hombres $(70,3 \%)$. La menor edad podría explicarse por el manejo preferentemente conservador (con limitación terapéutica) en los pacientes más añosos.

Se analizó la influencia de la edad en la severidad del trastorno deglutorio, realizando una regresión lineal, logrando marcar como punto de corte los 50 años. Al dividir a los pacientes en menores y mayores de 50 años, observamos que los pacientes más jóvenes presentaron un menor grado de severidad del trastorno deglutorio (1,4 vs $1,98, p=0,013)$, pese a presentar mayor frecuencia de intubación prolongada (50\% vs $27,1 \%)$. Nuestros datos nos permitirían afirmar que la menor edad ( $<50$ años) sería un factor protector en la evolución del trastorno deglutorio, pese a la mayor frecuencia de intubación prolongada, lo que se corresponde con lo descrito en otras series.

Para realizar la comparación entre patologías de ingreso y grados de severidad, se utilizó como grupo de referencia a los pacientes con AVE, por ser la patología que se presentó con mayor frecuencia, tal como se observa en otros trabajos ${ }^{7,8,15}$. De este modo destacan algunas patologías con mayor riesgo de trastorno de la deglución, tales como los pacientes con patología cardiovascular (tanto médica como quirúrgica), shock (de cualquier causa) y pacientes con enfermedades neurodegenerativas $(\mathrm{p}<0,05)$. Sin embargo, al corregir según el antecedente de intubación prolongada previa, esta asociación estadística desaparece, lo que reafirma la importancia de este antecedente en la evaluación de la deglución ${ }^{10}$.

Existen otras condiciones que, aunque contamos con un número pequeño de casos, son interesantes de analizar. Es el caso de los pacientes traqueostomizados $(\mathrm{N}=5)$, quienes presentaron un grado de severidad mayor que el grupo de referencia $(2,4$ vs 1,86$)$, similar a lo publicado por varios autores. Esto ocurriría por el impedimento anatómico para el ascenso laríngeo, así como la menor presión de aire en la subglotis, ambos fenómenos protectores durante la deglución ${ }^{17-19}$. 
Al contrario, los pacientes politraumatizados $(\mathrm{N}=5)$, presentaron un menor grado de severidad del trastorno deglutorio, pese a que todos habían tenido una intubación prolongada. Esto se podría explicar por su menor edad promedio $(37,2$ años) que, como vimos previamente, es un factor protector y por tanto, presentarían una evolución favorable, pese al antecedente de intubación prolongada ${ }^{20}$.

No realizamos análisis específico para el grupo de pacientes con cáncer de cabeza y cuello, por contar con escasos casos en esta cohorte $(\mathrm{n}=2)$. Sin embargo, en este grupo está descrita en la literatura la presencia de un mayor trastorno deglutorio, provocado tanto por la distorsión anatómica pre y postoperatoria, así como la disminución de la sensibilidad laríngea provocada por la quimioradioterapia ${ }^{19}$.

Finalmente, se logró realizar un seguimiento completo en $60 \%$ de los pacientes con trastorno moderado y $50 \%$ de los pacientes con trastorno severo de la deglución, observando que la gran mayoría de ellos logró realimentarse por vía oral antes de egresar del hospital. Las pérdidas en el seguimiento se deben a que no recibimos interconsultas para la reevaluación de los pacientes en todos los casos. Se puede observar que, a mayor severidad del trastorno deglutorio, menores fueron las posibilidades de egresar con alimentación por VO (78\% para los trastornos moderados, y 55\% para los severos, $\mathrm{p}=0,03)$, y mayor probabilidad de terminar con gastrostomía (GTT) como vía de alimentación al egreso hospitalario ( 5,6 vs $21,4 \%$, $\mathrm{p}=0,05)$. El tiempo de evolución de los pacientes a realimentación por $\mathrm{VO}$ fue de 8,7 días (rango 2 a 76) en los trastornos moderados, y de 14,3 días (rango 3 a 58) en los severos. El grado de severidad del trastorno deglutorio fue directamente proporcional con el número de días de evolución de los pacientes hasta alcanzar alimentación VO $(\mathrm{R}=48,9 \%, \mathrm{p}<0,001)^{21}$.

Uno de los objetivos que nos planteamos inicialmente fue intentar identificar precozmente a los pacientes que se beneficiarían de la indicación de GTT precoz. Sin embargo, no logramos identificar asociación a ninguna patología de base, género, ni antecedente de intubación previa. En general, fueron pacientes mayores que el resto del grupo estudiado (80 vs 71 años, $\mathrm{p}=0,018$ ), y $82 \%$ de ellos presentaba un trastorno severo de la deglución en su primera evaluación, lo cual reitera la necesidad de la evaluación seriada de los pacientes, para determinar el mejor momento y vía para su realimentación ${ }^{22,23}$.

Muchos pacientes con GTT siguen en control en nuestro equipo, y si logran una adecuada rehabilitación del trastorno deglutorio, se realimentarán por VO. Lo mismo ocurre con pacientes que egresan con sonda nasoenteral (SNG) que, según su evolución posterior, podrán realimentarse por VO o irán a GTT en los casos con mala evolución ${ }^{24}$.

\section{Conclusiones}

La evaluación nasofibroscópica de la deglución es simple, segura y confiable para la evaluación y el seguimiento de los pacientes con trastorno deglutorio.

La mayoría de los pacientes evaluados presentan un trastorno moderado o severo de la deglución $(65 \%)$, por lo que requieren postergar el inicio de la alimentación por $\mathrm{VO}$, en la mayoría de los casos.

La edad, y la historia previa de intubación prolongada son los antecedentes más importantes a considerar durante la evaluación de los pacientes con trastornos de la deglución.

Existen algunas patologías que se asociarían a mayor severidad del trastorno deglutorio. Sin embargo, al realizar una corrección estadística según el antecedente de intubación prolongada, estas diferencias desaparecen, por lo que se reafirma la importancia de este antecedente en la evaluación de la deglución.

La gran mayoría de los pacientes logra realimentación por vía oral antes de egresar del hospital. A mayor severidad del trastorno deglutorio, mayor demora en lograr realimentación por vía oral, y mayor riesgo de egresar con gastrostomía como vía de alimentación definitiva.

Este trabajo retrospectivo nos permite relacionar algunos factores de riesgo con el pronóstico del trastorno deglutorio en los pacientes que evaluamos diariamente. Un estudio de cohorte prospectiva permitiría obtener conclusiones con mayor validez.

\section{Referencias}

1. Johnson ER, McKensie SW, Sievers A. Aspiration pneumonia in stroke. Arch Phys Med Rehabil 1993; 74: 973-6. 
2. Marik PE, Kaplan D. Aspiration pneumonia and dysphagia in the elderly. Chest 2003; 124: 328-36.

3. Shapiro J. Evaluation and treatment of swallowing disorders. Comp Ther 200026 (3): 203-9.

4. Lind CD. Dysphagia: evaluation and treatment. Gastroenterol Clin N Am 2003; 32: 553-75.

5. Aguilera M, Torrejón M. Chile hacia el 2050: Proyecciones de población. Instituto Nacional de Estadísticas 2005, 16: 5-29.

6. Perry L, Love CP. Screening for dysphagia and aspiration in acute stroke. A systematic review. Dysphagia 2001; 16: 7-18.

7. Kumar S. Swallowing and dysphagia in neurological disorders. Rev Neurol Dis 2010; 7 (1): 19-27.

8. Martino R, Foley N, Bhogal S, Diamant N, Speechley M, Teasell R. Dysphagia after stroke: incidence, diagnosis, and pulmonary complications. Stroke 2005; 36 (12): 2756-63.

9. Skorets SA, Flowers HL, Martino R. The incidence of dysphagia following endotracheal intubation: a systematic review. Chest 2010; 137 (3): 509-10.

10. Barker J, Martino R, Reichardt B, Hickey EJ, RalphEdwards A. Incidence and impact of dysphagia in patients receiving prolonged endotracheal intubation after cardiac surgery. Can J Surg 2009; 52 (2): 119-24.

11. Barquist E, Brown M, Cohn S, Lundy D, Jackowski J. Postextubation fiberoptic endoscopic evaluation of swallowing after prolonged endotracheal intubation. A randomized, prospective trial. Crit Care Med 2001; 29: 1710-3.

12. Bours GJ, Speyer R, Lemmens J, Limburg M, de Wit R. Bedside screening tests vs videofluoroscopy or fibreoptic endoscopic evaluation of swallowing to detect dysphagia in patients with neurological disorders: systematic review. J Adv Nurs 2009; 65 (3): 477-93.

13. Ramsey DJ, Smithard DG, Kalra L. Early assessments of dysphagia and aspiration risk in acute stroke patients.
Stroke. 2003; 34 (5): 1252-7.

14. Beltrán M, Soler B, León N. Evaluación de la deglución a través de fibroscopia óptica. Rev Otorrinolaringol Cir Cabeza Cuello 2007; 67: 266-7.

15. Nazar G, Ortega A, Godoy A, Godoy JM, Fuentealba I. Evaluación fibroscópica de la deglución. Rev Otorrinolaringol Cir Cabeza Cuello 2008; 68: 131-42.

16. Arteaga P, Olavarría C, Naranjo B, Elgueta F, Espínola D. Cómo realizar una evaluación de deglución completa, eficaz y en corto tiempo. Rev Otorrinolaringol Cir Cabeza Cuello 2006; 66: 13-22.

17. Higgins D. Basic nursing principles of caring for patients with a tracheostomy. Nurs Times 2009; 105(3): 14-5.

18. Romero CM, Marambio A, Larrondo J, Walker K, Lira MT, Tobar E, et al. Swallowing dysfunction in nonneurologic critically ill patients who require percutaneous dilatational tracheostomy. Chest 2010; 137 (6): 1278-82

19. Manikantan K, Khode S, Sayed SI, Roe J, Nutting CM, Rhys-Evans P, et al. Dysphagia in head and neck cancer. Cancer Treat Rev 2009; 35 (8): 724-32.

20. Batty S. Communication, swallowing and feeding in the intensive care unit patient. Nurs Crit Care 2009; 14 (4): 175-9.

21. Squires N. Dysphagia management for progressive neurological conditions. Nurs Stand 2006; 20 (29): 53-7.

22. Gencosmanoglu R. Percutaneous endoscopic gastrostomy: a safe and effective bridge for enteral nutrition in neurological or non-neurological conditions. Neurocrit Care 2004; 1 (3): 309-17.

23. Kurien M, Sanders DS. Swallowing and dementiapractical solutions for a highly emotive problem? Clin Med 2010; 10 (3): 305-6.

24. Yokohama S, Aoshima M, Koyama S, Hayashi K, Shindo J, Maruyama J. Possibility of oral feeding after induction of percutaneous endoscopic gastrostomy. J Gastroenterol Hepato 2010; 25 (7): 1227-31. 